\title{
Spin and Translation, the Equation of Motion
}

\author{
Richard T. Hammond* \\ Department of Physics, University of North Carolina at Chapel Hill, and The Army Research Office, NC, USA
}

\begin{abstract}
The effect of spin on the equation of motion of a sliding object is assessed. The spatial dependence of the friction force on a spinning object is calculated and used to describe the equation of motion.
\end{abstract}

Keywords: Spin, equation of motion.

\section{INTRODUCTION}

The dynamics of a curve ball represents a well known example of the complex interactions between translation, rotation, and friction. A related example, which is finding a burgeoning range of applications, concerns the equation of motion of a sliding, rotating object such as a disk. When two objects slide along each other, there is often some component of rotation in addition to translation. The rotation adds complexity to the interaction, and there is no general approach that takes this into account in a systematic way.

Let us adopt the simplifying assumption that the magnitude of the kinetic friction force is proportion to the normal force. Then for an object either sliding without rotating, or rotating without sliding on a flat surface, the equation of motion is trivial. But if it is simultaneously rotating and sliding then the equation of motion becomes surprisingly complex.

A related effect was recently discussed that concerned a rotating sliding disk: It was shown that, due to rotation, the translation of a sliding object will end when the rotation does [1]. In their analysis they assumed that the normal force was constant, but also recognized that the normal force is not really constant, and deduced that the object would experience a sideways deflection. These authors also point out that there are important applications to packing and settling, and possibly to avalanches. In this paper I will derive this sideways deflection force and describe the equation of motion under certain approximations. This problem has also been discussed by Penner [2] where the application is the game of curling. In that work a functional form for the normal force is assumed whereas here the normal force is derived. Another interesting aspect of this problem is that, although we start with the assumption that the coefficient of sliding friction is independent of speed, the net frictional force is in fact a function of velocity. Velocity dependent frictional forces may also play an important part in the dynamics of sphere packing [3], exact solitonic

*Address correspondence to this author at the Department of Physics, University of North Carolina at Chapel Hill, and The Army Research Office, NC, USA; Tel: 919549 4313; Fax: 919549 4384;

E-mail: rhammond@email.unc.edu traveling wave solutions [4], nanotribological properties of $\mathrm{C}_{60}$ [5].

The effect is readily visible in many common lab or household situations and may be seen in sliding a cup or tumbler along a kitchen counter top, a kilogram mass (right cylinder) along a desktop, and of course many other similar situations. The effect was most pronounced when sliding a felt bottom chess piece (a pawn) along a polished dining room table.

\section{EQUATION OF MOTION}

The qualitative explanation is that the normal force on an object sliding with friction is not uniform, but is greater in the front (the direction of motion). Therefore, when looking at the friction component that arises from the spin it is evident that it is greater in the front than the back. This breaks the symmetry and produces a net force in the side wise direction, depending on the direction (up or down) of the spin.

To see the basic physics in a simple case, consider two small cylinders connected by a rigid massless rod- a squared off dumbbell, as shown in Fig. (1). Each cylinder has a height $2 b$, mass $m$, and are separated by a distance of $2 a$. Assume the "dumbbell" slides without rotating parallel to the rod. Then, assuming that the torque must vanish (otherwise it would flip up) one finds (See Appendix for details on derivation), taking the coefficient of kinetic friction to be $\mu$,

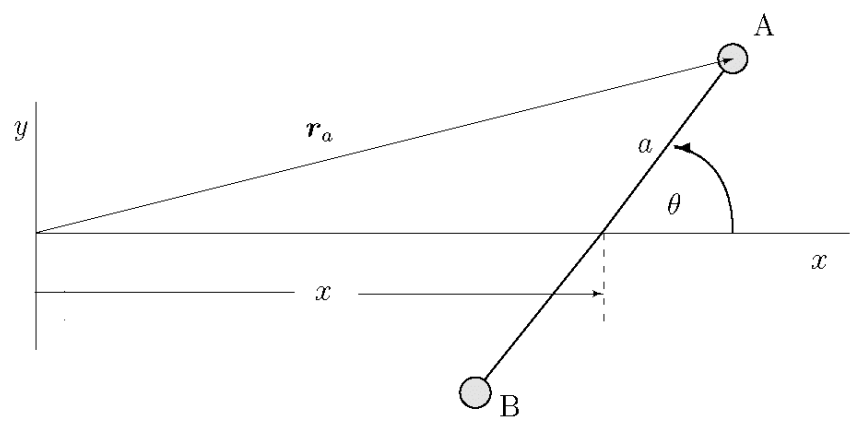

Fig. (1). The $z$ axis points up out of the page, so the counterclockwise rotation of the dumbbell has angular momentum in the postive $z$ axis. 


$$
\begin{aligned}
& N_{a}=m g\left(1+\mu \frac{b}{a}\right) \\
& N_{b}=m g\left(1-\mu \frac{b}{a}\right)
\end{aligned}
$$

where $N_{a}$ is the normal force on the front cylinder, cylinder A, and $N_{b}$ is the normal force on the rear cylinder, cylinder B.

Now suppose we consider the effects on an ordinary drinking tumbler. Before doing a detailed calculation one may place an upper bound on the sideways deflection by approximating the tumbler of radius $a$ by the dumbbell, and taking the $y$ component of the force to be $-\mu N_{a}+\mu N_{b}$, which gives a deflection of $-\mu k g t^{2}$ where $k=\mu b / a$. This is an over estimate because the force is distributed, and not all acting on $\pm a$, and also because the direction varies along the rim.

Now consider an instant when this object is rotating about its center of mass while the center of mass is translating in the $x$ direction. Let $x \hat{\boldsymbol{x}}$ be the coordinate to the center, $a \hat{\boldsymbol{a}}$ be a vector from the CM to the cylinder A, $-a \hat{\boldsymbol{a}}$ to the cylinder B, so that the positions of the cylinders are

$\boldsymbol{r}_{a}=\boldsymbol{x}+\boldsymbol{a}$

$\boldsymbol{r}_{b}=\boldsymbol{x}-\boldsymbol{a}$

and the velocities are

$\boldsymbol{v}_{a}=(U-a w \sin \theta) \hat{\boldsymbol{x}}+a w \cos \theta \hat{\boldsymbol{y}}$

$\boldsymbol{v}_{b}=(U+a w \sin \theta) \hat{\boldsymbol{x}}-a w \cos \theta \hat{\boldsymbol{y}}$

where $U=\dot{x}$ and $\omega$ is the angular velocity which points in the plus $z$ direction.

From now on the subscripts $a$ and $b$ will be dropped: cylinder A will be specified by $\theta$ and $\mathrm{B}$ by $\theta+\pi$, and if no parenthetical value of a quantity is explicitly given it, then it is taken to be a function of $\theta$. We can take the torque perpendicular to the direction of the rod in the $(x, y)$ plane to vanish and find (the derivation is in the Appendix)

$$
N(\theta)=m g \frac{1+\frac{k U \cos \theta}{v(\theta+\pi)}}{1+\frac{k U \cos \theta}{2}\left(\frac{1}{v(\theta+\pi)}-\frac{1}{v(\theta)}\right)} .
$$

To model a tumbler, which we assume has a bottom characterized by a thin ring of radius $a$, it is assumed that the frictional force from an element of the tumbler at $\theta$ is given by

$\Delta f=-\mu \Delta N \hat{v}$

where $\hat{\boldsymbol{v}}$ is the unit velocity vector, and $\Delta N$ is given by (5) with $m$ replaced by $\Delta m$ where $\Delta m=(m /(2 \pi) d \theta$. Thus the total frictional force on the tumbler is given by

$$
\boldsymbol{F}=-\frac{\mu}{2 \pi} \int \Delta N \hat{\boldsymbol{v}} d \theta
$$

In order to obtain an expression for the deflection in the $y$ direction, the $y$ component of this expression is considered next. The integration may be simplified if we restrict our attention to small $k$, which is usually valid for a wide variety of conditions (but not always) ${ }^{1}$. With this, $\Delta N$ is

$\Delta N(\theta)=\Delta m g\left(1+\frac{k U \cos \theta}{2}\left(\frac{1}{v(\theta+\pi)}+\frac{1}{v(\theta)}\right)\right)$.

The force in the $y$ direction is given by the $y$ component of (7), which, with (8), becomes after integration

$$
\begin{aligned}
& F_{y}=\frac{m g k \lambda}{2 \lambda^{2}\left(1+\lambda^{2}\right)}\left[\left(1+\lambda^{2}\right)^{2} E\left(\frac{4 \lambda^{2}}{\left(1+\lambda^{2}\right)^{2}}\right)\right. \\
& \left.-\left(-1+\lambda^{2}\right)^{2} K\left(\frac{4 \lambda^{2}}{\left(1+\lambda^{2}\right)^{2}}\right)\right]
\end{aligned}
$$

where $E$ is the complete elliptic integral of the second kind, $K$ is the complete elliptic integral of the first kind, and $\lambda=a \omega / U$.

Now let us consider the motion of the center of mass: We assume that, to lowest order, the coupling between translation and rotation is ignored, so that

$U=U_{0}-\mu g t$

$\omega=\omega_{0}-\mu g t / a$.

where $U_{0}$ is the initial velocity and $\omega_{0}$ is the initial angular velocity.

To the next order of approximation these are used in (9). I have not found an analytical expression for this integral so its numerical evaluation will be given. The results of course depend on various parameters, the initial translational speed $U_{0}$, the initial angular velocity $\omega_{0}$, the ratio of tumbler height to radius $b / a$, and as before the coefficient of kinetic friction $\mu$ (the mass $m$ of the tumbler drops away when the acceleration and its integrals are computed below). The most important of these is the value of $k(=\mu b / a)$ which contains the ratio of height to width. For a fixed radius, then the taller the tumbler, the bigger the effect is. This result follows from the same "common sense" notion that the taller (and narrower) the object is, the more likely it is to tip over. Essentially, what happens is that the ratio on the force at the front edge to the force on the back edge increases as the object gets taller and narrower. Typical values for a tumbler on a common black lab table are, $a=4 \mathrm{~cm}, \quad b=8 \mathrm{~cm}, U_{0}=100 \mathrm{~cm} \mathrm{sec}^{-1}$, $\mu=0.05$, and $\omega_{0}=v_{0} / a$. These numbers give the glass a two second time of flight, with the rotation ending at the same time as the translation.

Thus the velocity in the $y$ direction is given by

\footnotetext{
${ }^{1}$ Actually, the approximation is $\left(1+\frac{k U \cos \theta}{2}\left(\frac{1}{v(\theta+\pi)}-\frac{1}{v(\theta)}\right)\right)^{-1} \cong$ $\left(1-\frac{k U \cos \theta}{2}\left(\frac{1}{v(\theta+\pi)}-\frac{1}{v(\theta)}\right)\right)$. For the numerics used later this can be shown to be a good approximation.
} 
$v_{y}=\frac{1}{m} \int F_{y} d t$

and the displacement

$y=\int v_{y} d t$

A useful way to exhibit this is a parametric plot of $y v s$. $x$. Using (12) and $x$, found from integrating (10), this is shown in Fig. (2). Experiments performed on a common black lab table, also shown in Fig. (2).

The data was taken as was more of a reality check of the results than a proper physics experiment. A line was drawn across the glass with a marker and the initial angular velocity was obtained by measuring the angular rotation with a stop watch. In a better experiment this could easily be measured with a strobe light. The glass was started by hand, and only those "slides" that started along the $x$ axis were kept.

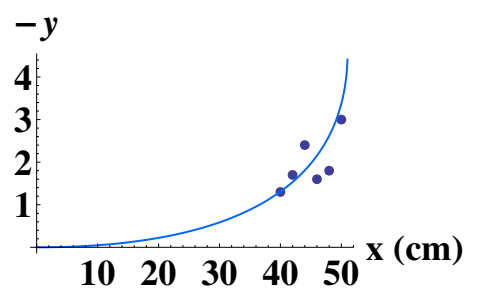

Fig. (2). The solid line is the theoretical result for the negative of the $y$ displacement $v s . x$ in $\mathrm{cm}$. The solid circles are the data of Tthe typical results of an experiment, measured in $\mathrm{cm}$.

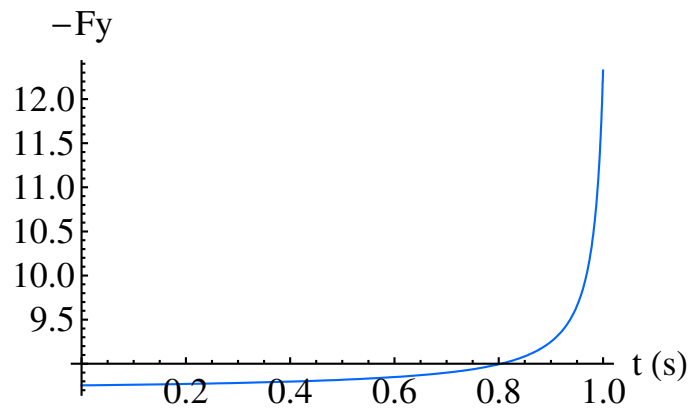

Fig. (3). The negative of $F_{y}$ vs. time in cgs units.

Another interesting aspect of the dynamics is that the deflection force increases slightly just before the tumbler comes to rest. This seems wrong at first glance, because the friction force is assumed to be independent of the magnitude of the velocity. But the friction force does depend, of course, on the direction and, as (8) shows, this enters into the resulting force. This is shown in Fig. (4), which is a plot of $F_{y}$ vs $t$ and shows that the deflective force increases somewhat as the tumbler nears the end of its motion. This is a direct result of the fact that the force increases with decreasing $\omega$, which is what Fig. (4) really shows.

Although the main thrust of this paper has been with the sideways deflection force, we can also look at the force in the $x$ direction, and demonstrate the non-obvious result that this forces decreases due to rotation, as first shown in [1].

From (7) we have

$F_{x}=-\mu m g I_{x}$ where

$$
\begin{aligned}
& I_{x}=\frac{1}{4 \pi} \int_{0}^{2 \pi} d \theta\left[2 \frac{(1-\lambda \sin \theta)}{v(\theta) / U}+k \frac{\cos \theta(1-\lambda \sin \theta)}{v(\theta+\pi) v(\theta) / U^{2}}\right. \\
& \left.+k \frac{\cos \theta(1-\lambda \sin \theta)}{v^{2}(\theta) / U^{2}}\right] .
\end{aligned}
$$

The integral may be evaluated in terms of elliptic integrals, but the graph is more illustrative and is shown in Fig. (5). For zero rotational velocity $\lambda=0$ and $I_{x}=1$ which shows that for an object under pure translation the frictional force in the $x$ direction is $-\mu m g$, as we know, but that as the rotational velocity increases the frictional force decreases.

\section{SUMMARY}

A simplified analysis of a sliding rotating object has been presented which shows how a sideways deflection force arises from the detailed interaction of translation and rotation. It is assumed that the friction is given solely by the coefficient of kinetic friction, which is assumed to be independent of the magnitude of the velocity. In reality, as the object comes to rest, the coefficient of kinetic friction must rise to the value of static friction. From this we can see that the actual coefficient of friction must be a function of the velocity, one that increases with decreasing speed.

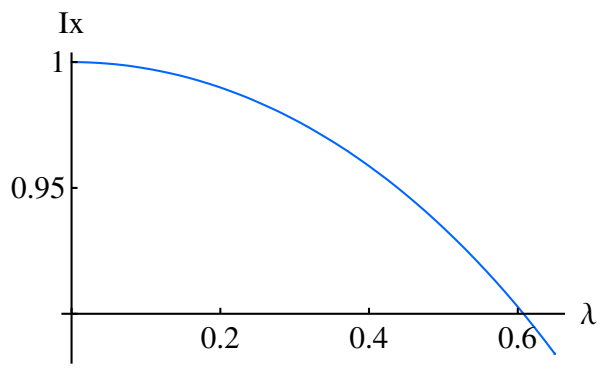

Fig. (4). This shows that the frictional force in the $x$ direction decreases with increasing rotational velocity $(\lambda=a \omega / U)$.

In addition, as the spinning tumbler comes to rest, one part of the tumbler may find itself at rest with respect the table momentarily. At this point the coefficient of static friction, which is substantially larger than the coefficient of sliding friction can dominate the final moments of the motion.

None of these effects is included in the theory, but at times their effects may be manifested in the experiments. Other variations are caused by the fact that the coefficient of friction along a typical surface is not really constant, even for clean lab table. In the case of the pawn and kilogram mass, where the deflection is considerably bigger, the coefficient of friction is larger, and, of course, the ring approximation fails and the method should be generalized to a disk surface.

\section{ACKNOWLEDGMENTS}

I would like to thank Mike Ciftan for helping me understand the nature of the friction force. 


\section{APPENDIX}

The object of this appendix is to derive (5). In order to do this it is necessary to discuss in more detail the nature of the model. The dumbbell is assumed to consist of two point masses, A and B. In a real dumbbell each mass would have some nonzero base area, and of course the normal force would vary throughout this area. In this case, assuming the dumbbell remains flat on the table, we would have to set the total torques in the $(x, y)$ plane equal to zero. Setting the torque along the axis perpendicular to the connecting rod axis to zero is a statement that the object does flip end over end style, and setting the torque along the axis to zero states that it does not "roll", or rotate around the rod axis. If we were really interested in a dumbbell, we would have to specify the width (measured perpendicular to the rod) of each cylinder, and compute the torque along the associated axis and insure that it does vanish. But we are using the dumbbell as a stepping stone to the tumbler, so we will stick with the point mass approach in which case we can only set the torque perpendicular to the connecting rod to zero.

To do this, we call the frictional forces on cylinders $\mathrm{A}$ and B $\boldsymbol{f}_{a}$ and $\boldsymbol{f}_{b}$ and assume they are $\mu$ times the normal force in magnitude, and opposed to the direction of the velocity so that.

The torque, $\tau$, through the center of mass about the axis perpendicular to the connecting rod arises from the normal forces and the friction forces. The component of the friction force on cylinder A along $\boldsymbol{a}$ is $\boldsymbol{f}_{a} \cdot \hat{\boldsymbol{a}}$, and similarly for the other friction force. Thus the torque is

$$
\tau=a\left(N_{a}-N_{b}\right)+b \hat{\boldsymbol{a}} \cdot\left(\mathbf{f}_{a}+\mathbf{f}_{b}\right)
$$

where $\mathbf{f}_{a}=-\mu N_{a} \hat{v}_{a}$ etc. Also, since each mass is $m$ we have

$N_{a}+N_{b}=2 m g$.

These two equations may be solved for $N_{a}$ and $N_{b} . N_{a}$ is found to be

$$
N_{a}=m g \frac{1+k \hat{\boldsymbol{a}} \cdot \hat{\boldsymbol{v}}_{b}}{1+\frac{k}{2} \hat{\boldsymbol{a}} \cdot\left(\hat{\boldsymbol{v}}_{b}-\hat{\boldsymbol{v}}_{a}\right)} \text {. }
$$

Now remember that $\hat{\boldsymbol{v}}_{a}=\boldsymbol{v}_{a} / v_{a}$ and use the notation that $v_{a}=v(\theta)$ and $v_{b}=v(\theta+\pi)$ and this result becomes (5).

\section{REFERENCES}

Farkas Z, Bartels G, Unger T, Wolf DE. Frictional coupling between sliding and spinning motion. Phys Rev Lett 2003; 90: 2483021.

[2] Penner AR. The physics of sliding cylinders and curling rocks. Am J Phys 2001; 69: 332.
[3] Silbert LE, Ertas D, Grest GS, Halsey TC, Levine D. Geometry of frictionless and frictional sphere packings. Phys Rev E Stat Nonlin Soft Matter Phys 2002; 65: 031304.

[4] Liang Q, Tsui OK, Xu Y, Li H, Xiao X. Effect of C60 Molecular Rotation on Nanotribology. Phys Rev Lett 2003; 90: 146102.

[5] Comte JC, Dinda PT, Remoissenet M. Discrete Burridge-Knopoff model, with exact solitonic or compactlike traveling wave solution. Phys Rev E Stat Nonlin Soft Matter Phys 2002; 65: 026615.

(c) Richard T. Hammond; Licensee Bentham Open.

This is an open access article licensed under the terms of the Creative Commons Attribution Non-Commercial License (http://creativecommons.org/licenses/by-nc/3.0/) which permits unrestricted, non-commercial use, distribution and reproduction in any medium, provided the work is properly cited. 